\title{
High Tumor Mutation Burden
}

National Cancer Institute

\section{Source}

National Cancer Institute. High Tumor Mutation Burden. NCI Thesaurus. Code C156025.

An indication that a tumor sample has a high number of genetic mutations per

megabase $(\mathrm{mb})$ of sequenced DNA. 